\title{
Gestion spatio-temporelle de la soudure alimentaire dans le Sahel burkinabé ${ }^{1}$
}

Paru dans la Revue Tiers-Monde, Paris, PUF, n¹80, octobre-décembre 2004, pp. 909-933.

\author{
Pierre JANIN \\ Géographe IRD \\ UR 106 "Nutrition, alimentation et sociétés" \\ Iedes - Université de Paris I \\ 45 bis, avenue de la Belle Gabrielle \\ 94736 Nogent sur Marne Cedex
}

Depuis les graves crises alimentaires des années 1970 et 1980, les pays sahéliens, ont, tour à tour, exploré diverses stratégies pour gérer le risque d'insécurité alimentaire à l'aide de systèmes de collecte de données régionalisés : planification de la redistribution de l'aide, stockage national de sécurité, prévisions pluviométriques, suivi satellitaire des cultures (Egg et Gabas 1997). Toutefois, ces approches ne dépassent généralement pas le cadre de la réponse immédiate à une situation d'urgence et ne permettent pas d'améliorer la gestion des disponibilités à l'échelle locale en élargissant l'horizon d'anticipation. Elles ne garantissent pas, non plus, un ciblage véritablement efficace de zones d'intervention et des populations à risque et ne disent également rien, la plupart du temps, sur les modalités réelles de répartition de l'aide alimentaire.

Qui plus est, à cette gestion étatique des situations d'insécurité alimentaire les politiques néolibérales sont en train de substituer, de facto, une gestion «décentralisée » - familiale et communautaire - de l'insécurité alimentaire. D'où l'importance des recherches susceptibles de mettre en évidence la capacité réelle des familles et des communautés villageoises à faire face à l'insécurité alimentaire conjoncturelle et structurelle (Hamelin et al. 1998), par une gestion efficace et combinée des stocks céréaliers et animaliers au cours de la soudure. Tout ceci impose, au préalable, l'identification des plus vulnérables à différentes échelles spatiales d'analyse en tenant compte de la variabilité intra-annuelle (la saisonnalité) et inter-annuelle (la récurrence) des disponibilités (Moseley 2001), dans un contexte aléatoire et incertain (Reardon et Matlon 1989). Car le temps - biologique de l'alimentation, descriptif des disponibilités et stratégique de la gestion - est une variable décisive aussi bien pour l'analyse des risques que pour la prise de décision.

En effet, faute de pouvoir anticiper, d'une année à l'autre, d'éventuels déficits alimentaires, la majorité des producteurs sahéliens doivent adopter une gestion prudente des stocks disponibles. La récurrence et la durée de la soudure alimentaire - exprimée par l'intervalle temporel entre la fin des réserves disponibles produites sur l'exploitation et la

\footnotetext{
${ }^{1}$ Ce texte s'appuie sur des données originales de terrain, collectées à l'échelles des concessions, des ménages et des individus selon les modules d'enquête, au cours de trois enquêtes saisonnières en milieu rural burkinabé réalisées, entre 2000 et 2002, dans le cadre d'un projet de recherche de l'IRD («Vulnérabilité alimentaire et sécurité nutritionnelle»), mené dans la province sahélo-soudanienne de la Gnagna (Est du Burkina Faso) qui constitue le prolongement septentrional du cœur historique du Gourma (de peuplement gourmantché).
} 
prochaine récolte céréalière (en nombre de jours) et quantifiée par l'ampleur du déficit entre besoins alimentaires et disponibilités réelles (en $\mathrm{kg} /$ personne/an) - renforce également l'importance stratégique de la gestion temporelle des réserves, à l'échelle familiale (greniers) ou villageoise (banques de céréales) (Makki 2001). C'est sur la base de cette définition première de la soudure alimentaire que s'enracinent les observations et les mesures réalisées, même si des déclinaisons plus complexes, souvent fluctuantes selon lesperceptions et les catégorisations imparfaites des intéressés, peuvent en être données ${ }^{2}$. Cette période est d'autant plus critique qu'elle se combine souvent, en milieu rural sahélien, avec un enclavement géographique en période d'hivernage, ce qui compromet fortement l'accessibilité aux marchés physiques. De fait, la gestion des disponibilités alimentaires sera d'autant plus aléatoire que le risque de déficit à la récolte sera récurrent et important. Toutefois, la prégnance de certains comportements oblatifs peut conduire à une gestion erratique des stocks lors des épisodes festifs et cérémoniels (funérailles par exemple) au risque d'aggraver l'insécurité alimentaire. De même, le besoin de liquidités monétaires peut amener certains producteurs à vendre leurs céréales sur pied à des collecteurs au risque de devoir en racheter à des prix élevés au cours de la soudure. Enfin, le producteur doit faire avec l'insécurité relative qui règne autour des réserves, en raison des vols ou des pertes au stockage.

Toute analyse de l'insécurité alimentaire implique donc de pouvoir évaluer, de manière quelque peu statique mais néanmoins précise, la situation alimentaire d'une unité géographique ou statistique (ménage, village,...), à la taille et la composition souvent fluctuante, à un moment donné. Compte tenu de la fragmentation des lieux de production et de stockage dans cet espace sahélien ouvert et du caractère fragmentaire des données secondaires disponibles, nous avons d'abord cherché à caractériser le risque alimentaire, c'est-à-dire la récurrence et l'imminence des déficits conjoncturels, par une lecture géographique du milieu, accordant une large place à un postulat plus déterministe que possibiliste. Par risque, en effet, nous avons choisi de prendre en compte une série de contraintes immédiates et d'aléas visibles, à la fois édictés par la nature et ses écarts saisonniers (aridité, instabilité, fertilité, hydrologie...) mais également résultant de déséquilibres liés à l'exploitation agro-pastorale des potentialités du milieu (densités rurales, saturation foncière, surexploitation des ressources) avec lesquels les groupes familiaux doivent composer, faute de pouvoir s'y soustraire et qui peuvent, qui plus est, se superposer spatialement et se recombiner dans le temps (Janin et Cambrézy 2003). Ces «risques apparents », sont autant d'indicateurs instantanés généralement utilisés par les « opérateurs » de développement lors de diagnostics participatifs. Une large place leur est donné dans ce texte même si progressivement les recherches de terrain montrent qu'ils sont à la fois peu fiables, aux échelles désagrégées, et ne permettent pas de rendre compte de la vulnérabilité réelle des individus et des groupes familiaux.

Par vulnérabilité alimentaire, on considère ici la sensibilité des individus, des familles, des communautés et des régions à l'insécurité alimentaire. Cette dernière dépend d'une exposition plus ou moins grande aux aléas et aux risques qui la commandent, comme d'une moindre capacité à mobiliser les ressources physiologiques, économiques, sociales ou écogéographiques de proximité ou lointaines pour y faire face (Courade 2001). A la différence des organismes d'appui intervenant à différentes échelles (Famine Early Warning System, Programme alimentaire mondial, Usaid, Fao, Catholic Relief Service,...), nous n'avons pas distingué une forme structurelle de vulnérabilité (évoluant peu ou lentement) et une vulnérabilité conjoncturelle (instabilité à court terme) étant donné les interactions temporelles

\footnotetext{
${ }^{2}$ En effet, il y a peu de points communs entre une situation de disette avérée sans recours au marché ou aux dons extérieurs faute de patrimoine ou de réseau relationnel et une période de soudure marquée par des achats massifs de céréales grâce à la mobilisation immédiate de revenus complémentaires.
} 
entre les différents déterminants de l'insécurité alimentaire. Cette analyse théorique de la vulnérabilité - qui associe les notions d'exposition, de sensibilité et de réactivité autour du risque - a ensuite été appliquée à l'approche spatio-temporelle de la soudure alimentaire que nous souhaitions mettre en œuvre sur le terrain. Des facteurs aggravants (qui renvoient à la notion de risque) ont été identifiés pour la région d'étude (la province agropastorale de la Gnagna). Mais c'est la question du temps qui a progressivement émergé, jusqu'à envahir l'ensemble du schéma conceptuel (fig. 1). Elle a été déclinée en fonction des temporalités de décision et d'action des acteurs familiaux (responsables de parcelle, gestionnaire de grenier, chef de ménage, propriétaire de troupeau). On distinguera ainsi trois composantes : les anticipations éventuelles, à court terme (pour les greniers) comme à plus long terme (pour la fertilité et la mobilité des hommes) qui permettent de réduire l'incidence de la soudure et ses conséquences négatives (déstockage et décapitalisation patrimoniale), les réponses instantanées aux situations de déficit alimentaire saisonnier - qui vont des achats alimentaires, à la diversification des revenus en passant par la vente de biens - et enfin les indicateurs avancés de dégradation des termes de l'échange (bétail/céréales) et de pression accrue sur les ressources alimentaires (volume de la ration de base). Ainsi théorisée, l'approche spatiotemporelle de la soudure proposée constitue un analyseur privilégié et complexe pour l'étude de la vulnérabilité réelle des groupes familiaux sahéliens - dans un contexte d'information fragmentaire et de leur capacité à faire face aux crises récurrentes.

Figure 1 : Schéma conceptuel de la soudure

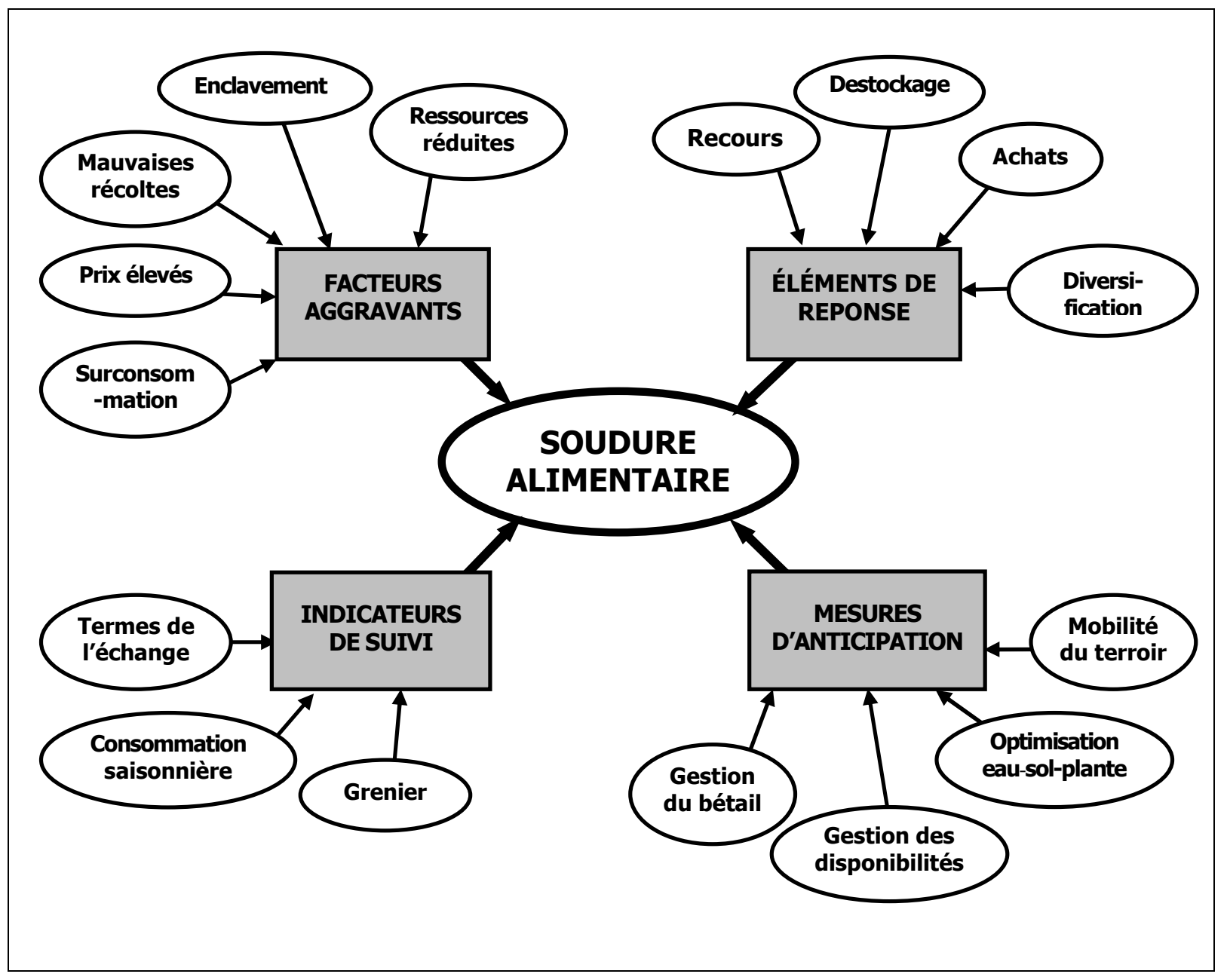


Ce texte répond donc à un double objectif : produire des connaissances, mobilisables par les intervenants de toute nature, pour rendre les communautés rurales moins vulnérables et moins dépendantes vis-à-vis des approvisionnements extérieurs en insistant sur la gestion opérationnelle des stocks mais aussi et surtout restituer au temps - qu'il soit descriptif, dans ses rythmes et ses accidents saisonniers; stratégique pour la réalisation des objectifs individuels et collectifs; ou encore systémique par le jeu des interrelations économiques, sociales et spatiales entre acteurs - la place centrale qu'il occupe dans le construit de la vulnérabilité, par les décisions et les actions des acteurs individuels qui engagent le devenir collectif des communautés familiales.

Après avoir présenté les approches méthodologiques développées en terme de diagnostic et de mesure de l'insécurité et les biais qu'elles entraînent, ce texte donne une large place à l'étude de la soudure alimentaire en milieu rural sahélien, dans ses mesures objectives (durée, besoins, disponibilités) comme dans ses perceptions subjectives. Il analysera ensuite les différentes stratégies de recours et les modes de gestion des réserves en période de soudure dont l'efficacité dépend à la fois du niveau des ressources mobilisables mais également des anticipations éventuelles.

\section{Le piège des indicateurs de risques apparents}

De nombreux organismes institutionnels ou privés (FEWS, PAM, FAO, USAID), travaillant à différentes échelles spatiales ont développé au cours de la décennie écoulée des méthodes d'analyse du risque afin de tenter de mieux gérer les effets des crises alimentaires en milieu sahélien. Leurs objectifs prioritaires - qui se recouvrent partiellement - visent à hiérarchiser les espaces en fonction du niveau de risque afin d'identifier les groupes de population les vulnérables pour permettre une meilleure allocation de l'aide alimentaire (Jaspers 1999).

Dans le cadre du projet de recherche «Vulnérabilité alimentaire et sécurité nutritionnelle » de l'IRD, une démarche similaire a, de prime abord, été adoptée. Les différents indicateurs conjoncturels de base (bilan céréalier, niveaux de prix, relevés pluviométriques), fournis par les systèmes nationaux ont été recueillis pour la province de la Gnagna (8.640 $\mathrm{km}^{2}$ ). Ils ont permis une première caractérisation objective du risque alimentaire à l'échelle régonale et donné lieu à la production de cartes régionales dont la figure 2 dresse une synthèse rapide.

Espace de transition, cette province longtemps enclavée se prête bien à une analyse spatiale des risques apparents et de ses effets (Ribot et al. 1996) : la partie septentrionale possède les caractéristiques des milieux sahéliens dégradés tandis que les empreintes paysagères de la moitié méridionale évoquent le domaine soudanien. Depuis une décennie, la pression sur les ressources (pâturage, bois, bas-fonds) s'accroît fortement sur fond de concurrence spatiale. Ainsi les communautés autochtones gourmantché, fortement majoritaires $(75 \%)$, doivent faire face à deux mouvements spontanés de «colonisation » agricole : mossi depuis le «Plateau central » surpeuplé à l'Ouest (5\% du peuplement), peul depuis les espaces sahéliens dégradés plus au nord (17\% du peuplement). Néanmoins aucune revendication ethno-communautaire d'importance n'a encore vu le jour, même si les litiges fonciers et pastoraux ne manquent pas (pour l'accès aux points d'eau, pour le passage des troupeaux en transhumance). Cette forme emblématique de confrontation pacifiée s'explique sans aucun doute d'abord par les liens de complémentarités noués entre pasteurs peuls et agriculteurs gourmantché dans un contexte de risques récurrents (cf. plus loin). Elle est également réactivée en permanence par un phénomène de mimétisme agro-économique peuls et gourmantché pratiquant indistinctement l'élevage, l'agriculture et la cueillette - mais 
surtout par des formes ritualisées d'échange (accueil, mariage, changement de noms et de prénoms, apprentissage linguistique réciproque). Cette province présente donc plusieurs traits marquants susceptibles d'en faire un cas d'étude intéressant et représentatif des sahels africains.

\section{Figure 2 : Niveaux de risque apparent}
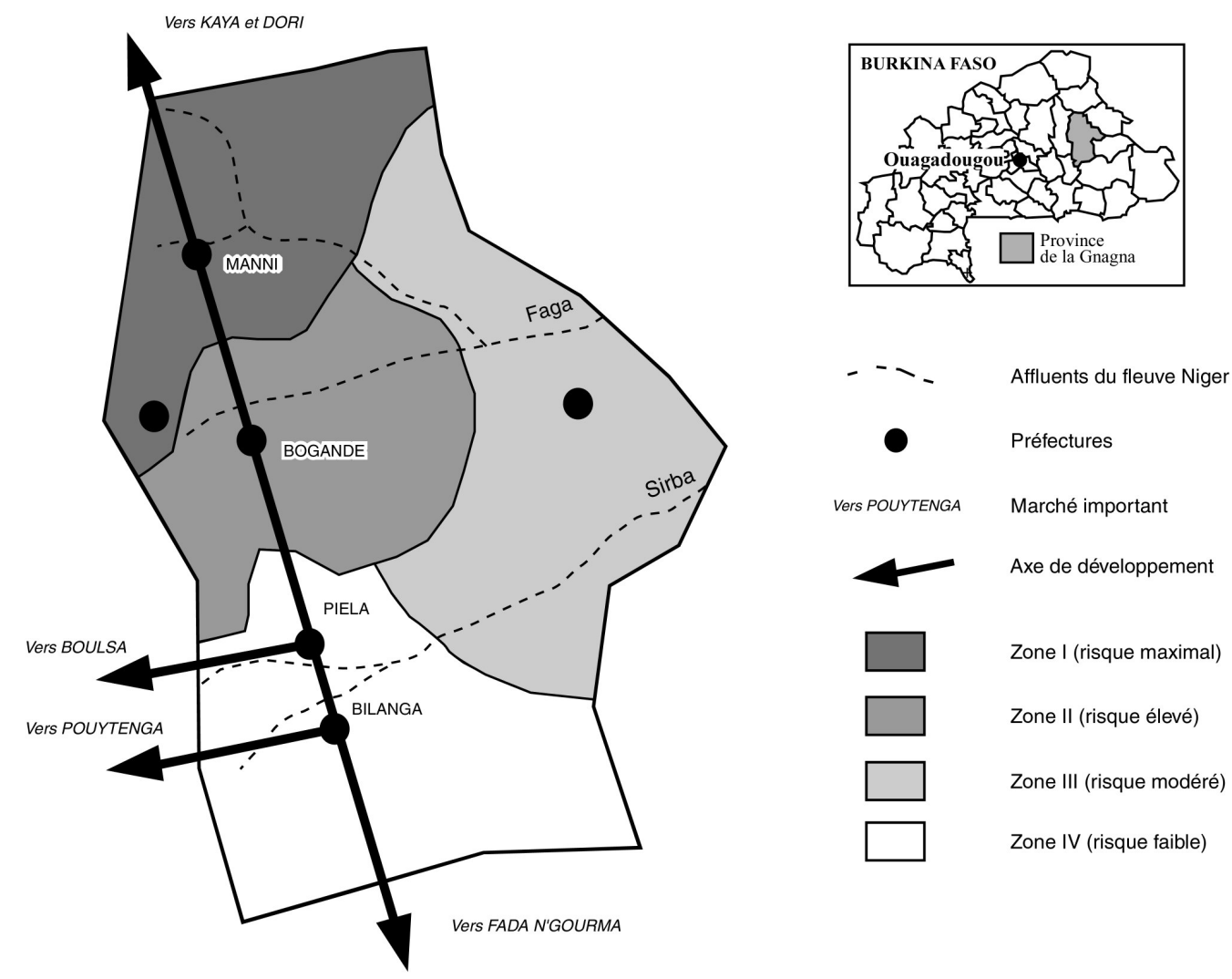

Zone I Zone II Zone III Zone IV

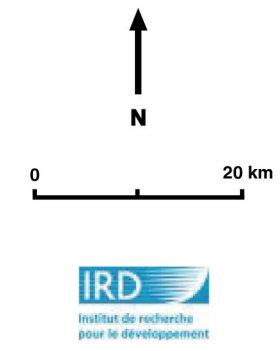

Source : enquêtes collectives Programme "VASN" Réalisation : P. JANIN (IRD)

La recherche a d'abord débuté par une enquête rapide en 2000 avec une « sélection » de personnes-ressources ${ }^{3}$ dans les 276 villages de la province. Des indicateurs de contraintes climatiques (instabilité et aridité) ou géographiques (enclavement), de gestion du milieu (densités, saturation foncière) ainsi que de sensibilité au phénomène de soudure ont été

\footnotetext{
${ }^{3}$ Chef de village, délégués administratif, représentant des communautés de migrants, responsable du comité villageois, villageois.
} 
recueillis au cours d'entretiens semi-dirigés collectifs (enquête 2000), complétés par des données secondaires. Après un premier dépouillement rapide des questionnaires, des cartes thématiques ont été établies pour chaque indicateur. Leur superposition a permis d'aboutir à une hiérarchisation des niveaux de risque apparent en quatre strates géographiques (Janin 2001 b et figure 2) selon l'intensité du phénomène observé (ce gradient est représenté à l'aide de croix dans le tableau ci-dessus). Puis, cette démarche a été systématisée afin de proposer un diagnostic territorial régionalisé (Ouedraogo 2001). In fine, cependant, ce sont les questions portant sur la soudure ${ }^{4}$ qui ont pesé le plus dans l'identification des zones de « risques apparents» (cf. fig. 2). Ce découpage géographique sera, par la suite, confronté avec les données recueillies auprès des ménages d'agropasteurs et croisées avec les données nutritionnelles collectées, auprès du même échantillon de personnes, par les chercheurs en nutrition du projet.

La première zone (de risque maximal) correspond au noyau dense de l'espace d'étude $\left(>80 \mathrm{hab} / \mathrm{km}^{2}\right)$. Les terroirs sont bien stabilisés et en voie de saturation foncière en raison de l'installation progressive de migrants agricoles mossi. Le taux annuel de croissance de la population y est, certes, élevé (autour de 3\%) mais reste nettement en deçà des autres zones. La pression pour l'accès et l'usage des ressources (terre, bois et eau) y est forte, même si la présence de plusieurs retenues d'eau a permis le développement de nouvelles activités agricoles (maraîchage, pêche, riziculture). Les sols y sont peu fertiles et fortement dégradés en raison de la disparition progressive de la jachère et de la coupe accélérée de bois de chauffe. Si les variations pluviométriques entre la partie septentrionale et méridionale ne sont pas spectaculaires (100 millimètres en moyenne sur quatre séries décennales), le caractère sahélien du climat apparait plus affirmé (l'indice annuel d'aridité est supérieur à 2,8). Les villages sont peu enclavés tandis que les relations d'échange marchand, plus développées, permettent de dégager des ressources monétaires supplémentaires (embouche animale, petit commerce).

La deuxième zone limitrophe (de risque aggravé) occupe une plus grande superficie. Si les types de risques et les contraintes ne diffèrent pas sensiblement avec le sous-espace précédent, en revanche, elles ne revêtent pas la même intensité. Les densités rurales sont moins élevées (40-60 hab/ $\left.\mathrm{km}^{2}\right)$ mais restent supérieures à la moyenne provinciale $\left(37 \mathrm{hab} / \mathrm{km}^{2}\right.$ en 1998). L'accès à la terre reste encore possible pour des allochtones même si la multiplication des hameaux de culture constitue un indicateur patent de saturation progressive. Toutefois, une forte majorité d'exploitants parviennent à maintenir une période de jachère entre les rotations culturales. La variabilité intra-annuelle des précipitations s'estompe quelque peu (indice $<2,5$ ) tout en restant plus élevée que dans le secteur méridional sahélo-soudanien. L'enclavement géographique ne touche pas l'ensemble des villages enquêtés mais s'impose comme une contrainte saisonnière.

Dans les deux dernières zones, la compétition pour l'accès et l'usage de la ressource foncière ou ligneuse ne revêt pas la même intensité. Des potentialités non négligeables (terre, bois, eau) perdurent étant donné la charge démographique réduite (densités $<20 \mathrm{hab} / \mathrm{km}^{2}$ ). Toutefois, le rythme annuel de croissance démographique est nettement supérieur aux autres zones (6\% contre 3\%). En contrepartie, les activités traditionnelles d'élevage, transhumant ou non, sont plus développées compte tenu de la présence accrue d'agro-pasteurs peuls, plus ou moins sédentarisés. L'indice d'aridité décroît sensiblement (proche de 2) en raison d'une pluviométrie sensiblement supérieure : $650 \mathrm{~mm}$ annuels moyens depuis 1960 contre $550 \mathrm{~mm}$

\footnotetext{
${ }^{4}$ «Les greniers étaient-ils vides en début de saison des pluies ? Avez-vous emprunté des céréales ? Consommezvous fréquemment des feuilles de cueillette pour vous nourrir ? A quel prix moyen avez-vous acheté le yorouba de mil ou de sorgho après récolte et en début d'hivernage ?».
} 
dans la première zone. De ce fait, la variabilité inter-annuelle des prductions se réduit sensiblement : les déficits céréaliers sont à la fois moins importants et moins fréquents.

$\mathrm{Si}$ ce diagnostic se révèle pertinent pour une intervention, il révèle toutefois ses limites: la variabilité spatiale intra-zonale n'est pas prise en compte de même que la dynamique du système alimentaire et la différenciation ethno-communautaire. Il s'appuie fortement sur des données secondaires dont la fiabilité n'est pas toujours prouvée ou sur des indicateurs instantanés agrégés à l'échelle du village. Trop descriptif et statique, il n'intègre pas certains phénomènes majeurs en milieu rural sahélien tels que l'effet de la saisonnalité, la succession des crises, l'inégale vulnérabilité des familles et des individus. C'est pourquoi, une série d'enquêtes saisonnières coordonnées, à différentes échelles (concession, ménage, individu) visant à mesurer le niveau d'insécurité réelle et la capacité collective des acteurs à y faire face, a été mise en place.

\section{La difficile mesure de l'insécurité réelle}

Comment rendre compte de la réalité de déficits alimentaires fluctuants, émiettés et masqués ? Comment appréhender la soudure à la fois récurrente et aléatoire ? C'est à ce dilemme méthodologique que nous avons été confrontés en parcourant cette province rurale. Le caractère semi-dispersé de l'habitat collectif ${ }^{5}$ constitue une première contrainte. Le village gourmantché - qui égrène ses concessions rurales sur plusieurs kilomètres, comme dénué de toute centralité - échappe à l'observateur le plus exercé. D'autant plus que les terroirs imparfaitement figés, évoluent au gré des défrichements et de la formation de hameaux de culture où résident durant plusieurs mois une partie du ménage. Cette multipolarité résidentielle renvoie à la saisonnalité d'un mode de vie organisé autour des travaux agricoles et rythmé par les pluies d'hivernage. A l'émiettement extraordinaire des lieux de stockage céréaliers qui en résulte - on relève des greniers de case, de cour, de champ, de brousse ou de hameau de culture - répond également la multiplicité des modes d'appropriation et de gestion qui ne se recouvrent qu'imparfaitement. De prime abord, cette distinction résiste à l'analyse puisque l'individu peine à exister en dehors du groupe familial dont il dépend fondamentalement pour sa survie. Et pourtant, de même que les enquêtés distinguent aisément «champ collectif » et « parcelle individuelle », émergeront peu à peu, au cours des enquêtes, des greniers collectifs de famille, des greniers collectifs de ménage, des greniers collectifs de couple, des greniers pluri-individuels et des greniers individuels. Cette différenciation ne répond pas à un simple besoin classificatoire mais bien au souci de rendre compte de l'enchevêtrement des centres de décision au sein des ces petites communautés spatiales. De fait, l'appropriation effective des greniers joue sans doute un rôle moins déterminant dans la lutte contre l'insécurité alimentaire que les droits efficients de gestion et l'enchaînement temporel des décisions ${ }^{6}$.

Ainsi, les greniers constituent à la fois des lieux stratégiques de stockage des réserves mais également un véritable espace social où se déploient des micro-stratégies de pouvoir, illustrant le caractère inégalitaire des régulations mises en oeuvre qui s'enracinent à la base à la fois sur des disparités de statut (âge et sexe) mais également sur des inégalités

\footnotetext{
${ }^{5}$ Chaque concession rurale gourmantché est organisée selon un schéma spatial dominant même s'il tend à évoluer avec la segmentation lignagère et le phénomène marqué d'individualisation progressive des ménages. Elle est formée par plusieurs cases individuelles en banco (terre), entourées par un mur d'enceinte, autour d'une cour centrale, à la fois point de centralité géographique et espace social privilégié qui met en scène le un système familial multipolaire, constitué de plusieurs centres de production, de consommation et de décision ayant des relations complexes, structurées et évolutives en terme d'échange alimentaire (aide, solidarité, préparation).

${ }^{6}$ Décision d'ouverture, de fermeture, de report d'utilisation, de redistribution, de vente.
} 
patrimoniales manifestes. D'où la nécessité de dresser un bilan exhaustif des disponibilités alimentaires individuelles mais également des ressources supplémentaires mobilisables (bétail, argent) en cas de déficit, avant d'agréger ces données à l'échelle du ménage. C'est ce minutieux travail qui a été réalisé en 2001 et 2002 auprès de quelque 1400 greniers et 2000 parcelles de culture, à partir d'un échantillon aléatoire de 180 Unités Collectives d'Habitat représentant environ 625 ménages réparties dans les quatre zones de risque apparent ${ }^{7}$. Pour évaluer le niveau des stocks disponibles, si plusieurs méthodes existent ${ }^{8}$, une seule a retenu notre attention : la mesure individualisée des greniers de mil et de sorgho après-récolte, associée à une visite des parcelles cultivées, permettant de vérifier les informations. Après calcul du volume réel des céréales stockées, un coefficient moyen, tenant compte du type de céréale, de la qualité du grain et de la densité de stockage, a permis de convertir ces volumes en kilos-équivalent céréales.

\section{Figure 3 : Exemple de chronogramme mensualisé}

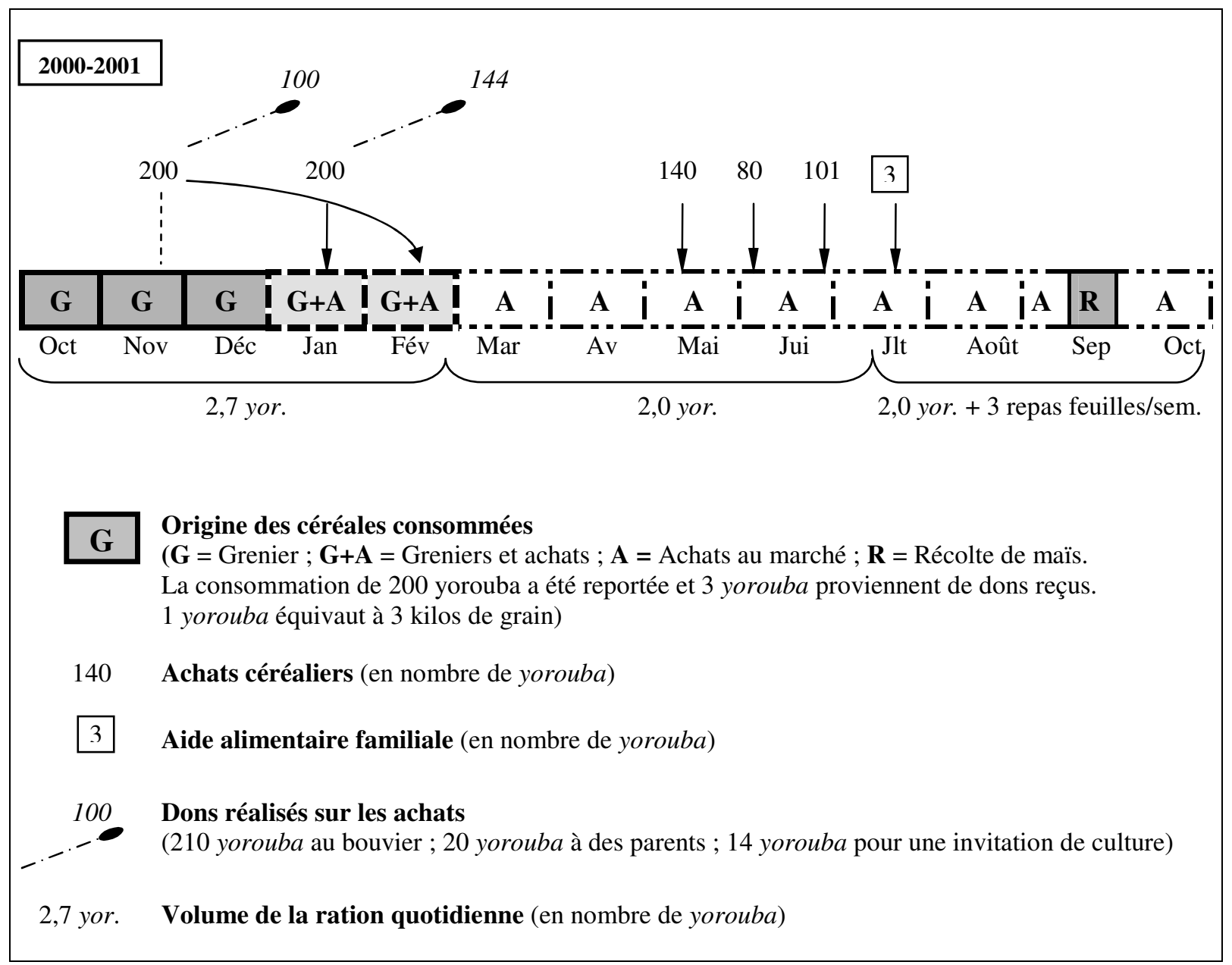

\footnotetext{
${ }^{7}$ Le choix des concessions rurales a été effectué par tirage au sort après établissement de la liste des ménages, sur la base des cahiers administratifs villageois datant de 1998.

${ }^{8}$ La plus couramment utilisée consiste à extrapoler la valeur des récoltes à l'aide de la méthode des carrés de rendement. Une autre s'appuie sur les déclarations des actifs pour estimer le volume des disponibilités alimentaires effectivement réservées à la consommation (Janin 2001a).
} 
A ce stade, cette méthode n'apporte que peu d'éléments d'information sur les mécanismes de redistribution interne au groupe familial et sur les modes de gestion des stocks au cours de l'année. C'est pourquoi, un suivi saisonnier de chaque grenier a été réalisé - à chaque période critique : «après récolte » lorsque les disponibilités sont abondantes ; «en saison sèche» lorsque les premières difficultés alimentaires apparaissent; et en « hivernage » lorsque les déficits imposent des ajustements quantitatifs et qualitatifs et la mise en place de stratégies de recours - sur la base des déclarations de chaque responsable in situ avec un récapitulatif en fin d'année.

Les événements-clés de la gestion temporelle de chaque grenier (ouvertures et fermeture) ont, ensuite, été reportés sur un chronogramme mensualisé synthétique (fig. 3). Ce dernier a progressivement été enrichi et complexifié par l'adjonction de nouveaux marqueurs temporels de consommation (ration journalière moyenne, fréquence de préparation des feuilles de cueillette). Enfin, à l'aide des modules d'enquête consacrés aux «stratégies de recours en période de soudure », nous avons fait figurer les différents épisodes d'achats céréaliers immédiats ou anticipés, les décisions de report de consommation, le volume de l'aide reçue ou octroyée. Cette représentation graphique, au-delà de l'information visuelle immédiate qu'elle délivre sur l'intensité et la durée de la soudure, offre l'insigne avantage de recouper l'ensemble des informations disparates afin de dresser, pour chaque ménage, un bilan alimentaire agrégé, relativement fiable.

Dans le cas présenté, les céréales stockées dans les greniers ont été rapidement consommées : dès le mois de janvier, le chef de ménage choisissait d'alterner la préparation du sorgho produit sur l'exploitation avec le mil acheté sur le marché. Il avait, certes, anticipé l'achat de plusieurs sacs de céréales (400 yorouba) afin de bénéficier de prix moins élevés mais, fort du soutien alimentaire procuré au peul chargé de la surveillance et de l'entretien du cheptel bovin transhumant (210 yorouba), ce complément a été épuisé dès le mois d'avril, nécessitant trois réapprovisionnements successifs (140, 80 et 101 yorouba). Mais ce recours au marché s'est révélé insuffisant pour maintenir le niveau de la ration céréalière de base $(2,7$ yorouba), rapidement réduite à 2,0 yorouba et bientôt comlétée par la consommation de feuilles de cueillette. Ces différents ajustements n'ont cependant rien d'exceptionnel en milieu rural sahélien.

Il était néanmoins présomptueux de caractériser le niveau d'insécurité et de vulnérabilité d'un ménage à l'aide d'une seule année d'observation étant donné la variabilité annuelle des disponibilités alimentaires et des profils de gestion. C'est pourquoi, cette analyse détaillée a été reconduite en 2002 avec les mêmes chronogrammes. Ainsi progressivement, le temps est devenu un facteur-clé d'analyse et nous avons cherché à lui donner toute sa mesure : temps immédiat de la consommation, temps moyen des anticipations de gestion des greniers et temps plus long des effets annualisés des déficits alimentaires.

Par ailleurs, si des réserves céréalières abondantes sont de nature à réduire le risque d'insécurité alimentaire, c'est-à-dire la durée de la soudure et l'ampleur des déficits, elles ne constituent malheureusement pas un indicateur toujours probant de leur mobilisation future pour la consommation familiale, car les dons, les ventes et la surconsommation lors des épisodes festifs sont susceptibles de les amoindrir. De même, des revenus monétaires supérieurs à la moyenne ne se traduiront pas nécessairement par une meilleure accessibilité économique aux aliments dans la mesure où les termes de l'échange entre bétail et céréales sont instables et volatils. 


\section{Les écarts temporels et les discontinuités spatiales de la soudure}

Le caractère itératif de la soudure alimentaire en milieu rural sahélien sur fond de potentialités amoindries et de pauvreté endémique est de nature à conforter certaines analyses déterministes. Ainsi, ces «milieux » sont encore souvent considérés comme homogènes, tant du point de vue des contraintes bio-climatiques (fortes), des potentialités (faibles), que des modes de production (considérés comme "traditionnels »). En réalité, il existe une grande variabilité spatio-temporelle des catégories et des espaces à risque (Raynaut et al. 1998, Courade et al. 2001). Mais ce phénomène, relativement connu, reste toujours difficile à quantifier et modéliser à petite échelle. Ainsi, les surplus départementaux peuvent cacher des déficits locaux tandis la présence de nombreux greniers collectifs est loin de signifier que tous les ménages et les dépendants y ont accès pérenne. Ceci explique l'intérêt des transferts d'échelle tant géographique que statistique pour mieux appréhender la vulnérabilité alimentaire réelle. A tel point qu'une disette marquée, suivie d'une «bonne» année agricole aura moins de conséquences négatives qu'une succession d'années agricoles médiocres obérant toute possibilité de reconstitution des stocks céréaliers et des ressources monnayables (outil, cheptel, épargne).

Les enquêtes de terrain, réalisées entre 2000 et 2002, ont confirmé la prévalence élevée de la soudure : 70 à $90 \%$ des ménages ruraux sont touchés et ne parviennent presque jamais, malgré d'importants ajustements de la ration alimentaire à atteindre les prochaines récoltes. Ainsi, la soudure semble faire partie de l'ordre banal des choses au même titre que l'irrégularité pluviométrique, la pauvreté et la maladie. Elle n'est donc pas toujours véritablement perçue comme un risque quantifiable et prévisible par les actifs agricoles, faute de pouvoir élaborer de recours stratégiques efficaces à moyen terme. Au-delà d'un discours plutôt uniformisant, si l'on excepte les années de crise marquées par des décès ou un amaigrissement généralisé, une fine analyse temporelle montre que la soudure est marquée par des écarts temporels et d'évidentes discontinuités spatiales. Ce semblera quelque peu paradoxal à certains puisque les conditions (sol, eau) comme les facteurs de production (travail, outillage, fumure) restent sensiblement les mêmes.

Ainsi en raison d'un «bon hivernage» (saison des pluies) - la pluviométrie se rapprochant de la moyenne décennale 1960-1969 $(685 \mathrm{~mm})$ - les récoltes céréalières de 1999 ont été relativement abondantes ${ }^{9}$. C'est pourquoi, en fin de saison sèche 2000, la disette ne touchait que $40 \%$ des villages de la Gnagna avec une prévalence géographique très variable selon les zones identifiées : plus élevée dans la partie sahélienne (60\%), faible ailleurs (10 à 20\%). Deux indicateurs complémentaires instantanés venaient également confirmer cette disparité géographique : l'aide alimentaire institutionnelle et la préparation alimentaire de feuilles de cueillette (balanitès, fédou) étaient nettement plus fréquents au nord qu' au sud ${ }^{10}$.

Les deux «années alimentaires ${ }^{11}$ suivantes ont été très contrastées : 2000-2001 a été marquée par des déficits précoces et aggravés avec des fortes disparités géographiques entre zones et une forte variance intra-zonale tandis que 2001-2002 était caractérisée par des déficits temporels et quantitatifs moins importants et une moindre hétérogénéité spatiale (tableau 1). Quels sont les éléments susceptibles d'expliquer cette disparité ? A l'échelle

\footnotetext{
${ }^{9}$ En revanche, des pertes avant récolte très importantes ont été observées dans les parcelles de bas-fonds en raison de la durée inhabituelle d'ennoyage des tiges. Ce phénomène semble essentiellement lié à l'augmentation globale des écoulements de surface sur l'ensemble des affluents du fleuve Niger.

${ }^{10} 60 \%$ des villages de la zone I, contre $30 \%$ en zones II et III et $15 \%$ en zone IV.

${ }^{11} \mathrm{~L}$ ' « année alimentaire » diffère sensiblement de l'année agricole (qui démarre en juin avec les semis) : elle débute en novembre après récolte pour s'achever à l'automne de l'année civile suivante.
} 
régionale, le facteur climatique n'est pas à écarter mais il reste peu probant pour valider les inégales disponibilités des ménages appartenant à une même concession. Néanmoins, la diminution progressive des précipitations annuelles, comme l'atteste les séries chronologiques depuis 1960, constituerait un aléa moins grand que leur répartition aléatoire au cours de la saison.

Tableau 1 : Nombre moyen de jours de soudure (par ménage)

\begin{tabular}{|c|c|c|c|c|c|}
\hline & Zone I & Zone II & Zone III & Zone IV & Moyenne \\
\hline 2001 & 175 & 210 & 187 & 155 & 185 \\
\hline 2002 & 107 & 117 & 118 & 126 & 115 \\
\hline \multicolumn{6}{c}{ Source : IRD/Univ. Ouagadougou/IEDES-Paris I, 2001 et 2002.}
\end{tabular}

L'hivernage 2000 n'a pas connu de déficit pluviométrique aggravé - les hauteurs d'eau restant proches des normales décennales 1990-1999 (500 mm) - mais il a été marqué par un arrêt brutal des précipitations en août, suivi par de brefs mais violents épisodes orageux (143 $\mathrm{mm}$ en une journée notamment), dont les conséquences ont été marquées sur la maturation des épis et, par suite, sur les rendements. De ce fait, le volume des céréales récoltées à l'automne 2000 était nettement inférieur à celui de la campagne 1999. Ceci explique la durée moyenne exceptionnelle de la soudure 2001, avec une répartition atypique puisqu'elle dépassait 190 jours pour la moitié des ménages enquêtés. Elle était également précoce puisque $40 \%$ des ménages interrogés étaient déjà frappés au mois de mars et $67 \%$ en juillet. Contre toute attente, en dépit de la pénurie généralisée en milieu paysan, l'aide alimentaire était presque «invisible », mal répartie et inefficace étant donné sa modicité (1 à 3 yorouba pour les ménages les plus chanceux) et les détournements «sociaux» ou frauduleux. Par comparaison, le bilan que l'on peut dresser de la soudure 2002 est nettement plus favorable. A peine $10 \%$ des enquêtés avaient des greniers vides en mars et $36 \%$ en juillet. Les écarts temporels et les discontinuités spatiales ont été moindres, toutes les zones d'enquête ayant une soudure moyenne comprise entre 105 et 130 jours. Par ailleurs, sa prévalence était sensiblement moins élevée (71\%).

En réalité, appréhender le phénomène de la soudure par la mesure des déficits agrégés ou reportés paraît quelque peu réducteur, car cette période critique pour les ménages ruraux est également marquée par une tension croissante entre l'offre et la demande, non seulement pour les céréales localement consommées (mil, sorgho) mais également les céréales importées (maïs, riz) et pour le bétail. Au cours de cette période - qui commence parfois dès le mois de décembre pour s'achever en septembre avec les récoltes du maïs - la dépendance des ménages ruraux par rapport au marché (Adger 1999) est forte, même dans les villages les plus reculés, puisque plus de $85 \%$ des céréales consommées au cours de la soudure proviennent des achats réalisés sur le marché. Et sur l'ensemble de l'année, la part des achats reste très élevée en année déficitaire et non négligeable en année « moyenne » (tableau 2 ci-dessous).

Tableau 2 : Nombre de yorouba achetés par ménage (et en \% des besoins annuels)

\begin{tabular}{|c|c|c|c|c|c|}
\hline & Zone I & Zone II & Zone III & Zone IV & Moyenne \\
\hline 2001 & $350(46,1)$ & $323(53,3)$ & $343(52,5)$ & $272(38,0)$ & $329(48,2)$ \\
\hline 2002 & $223(26,5)$ & $199(31,0)$ & $207(28,5)$ & $235(29,4)$ & $214(28,5)$ \\
\hline
\end{tabular}

Source : enquêtes IRD/Univ. Ouagadougou/IEDES-Paris I, , 2001 et 2002 
L'intensité et la gravité de la soudure dépend donc fortement de la capacité économique des ménages, en situation de pénurie alimentaire, à acquérir des denrées sur les marchés physiques. De ce fait, les fluctuations saisonnières des prix constituent à la fois des indicateurs avancés et instantanés de la soudure, sur lesquels s'appuient les commerçants et les agriculteurs les plus prudents pour anticiper les décisions d'achat de céréales et de vente de bétail. Ainsi en 2001, compte tenu des ces comportements, le prix du yorouba après récolte (310 francs CFA en novembre) était déjà nettement supérieur à celui des années où le phénomène de soudure est peu marqué tandis que l'envolée des prix se poursuivait pour atteindre des niveaux très élevés en juillet (565 francs CFA).

Tableau 3 : Prix minima et maxima d'achat du yorouba de mil en francs CFA sur la base des déclarations individuelles (et \% de variation)

\begin{tabular}{|c|c|c|c|c|c|}
\hline & Zone I & Zone II & Zone III & Zone IV & Moyenne \\
\hline 2000 & $150 / 255(69)$ & $150 / 220(50)$ & $125 / 215(71)$ & $145 / 200(40)$ & $145 / 230(57)$ \\
\hline 2001 & $300 / 580(92)$ & $335 / 570(70)$ & $310 / 555(78)$ & $300 / 530(77)$ & $310 / 565(81)$ \\
\hline 2002 & $265 / 500(90)$ & $260 / 495(91)$ & $240 / 493(83)$ & $270 / 500(85)$ & $260 / 500(93)$ \\
\hline
\end{tabular}

Dans le même temps le prix du bétail - qui constitue la principale ressource monnayable pour les agropasteurs gourmantché et peul de la région - connaissait une forte dépréciation en raison d'une offre surabondante sur le marché. Cette évolution corrélée des prix des céréales et du bétail a eu pour effet d'accroître la vulnérabilité des ménages ruraux, incapables de maintenir leur effort d'achat. Par conséquent, si les caractéristiques objectives de la soudure (durée et intensité) fournissent des indications intéressantes sur l'exposition des ménages au risque d'insécurité alimentaire, elles s'avèrent impuissantes à rendre compte de leur vulnérabilité et doivent être confrontées à des marqueurs temporels de gestion.

\section{La période stratégique de la soudure}

La période de la soudure est stratégique à plus d'un titre. D'abord parce qu'elle révèle la nature conflictuelle et hiérarchique des rapports sociaux les plus immédiats en situation de pénurie qui se donnent à lire dans les arbitrages, les préférences et les exclusions éventuelles. En ce sens, elle prouve et met à l'épreuve les liens intra-communautaires et extracommunautaires. Ensuite, parce qu'elle constitue un moment privilégié pour évaluer et comparer l'efficacité de la réponse sociétale en matière de gestion des ressources, avec des résultats très ambivalents selon les années et la modulation temporelle des décisions.

Par ailleurs, mais nous n'aurons pas l'occasion de le développer, l'étude de la soudure met en exergue le rôle déterminant des réseaux marchands en matière d'approvisionnement alimentaire et le poids des relations d'échanges marchands dans des espaces où l'État et les institutions ne sont pas toujours en mesure d'anticiper les crises éventuelles. Ainsi, les commerçants de céréales et de bétail (ces deux spéculations étant généralement associées) ont l'insigne avantage d'être durablement installés dans cet espace, de disposer de magasins décentralisés dans de nombreux petits marchés ruraux et de pouvoir maîtriser l'accès à une information fragmentaire ; ils ont, de ce fait, une réactivité bien supérieure aux intervenants institutionnels pour répondre à une demande évolutive et dispersée. Inversement, pour les familles d'agropasteurs, faute de pouvoir augmenter leurs productions céréalières, la lutte la plus efficace contre l'insécurité alimentaire passe par un recours accru au marché. 
Enfin, la période de soudure est stratégique parce que si les déficits alimentaires ne sont pas une fatalité ils restent cependant fréquents et prévisibles (Gado 1993) et engagent durablement l'avenir immédiat de chaque groupe familial. Les seules incertitudes portent sur sa durée et la capacité des individus et des ménages «à passer la soudure ». Un bref rappel des soudures passées a ainsi permis d'établir qu'environ $40 \%$ des villages de la Gnagna ont connu deux disettes au cours de la décennie écoulée et $10 \%$ plus de trois épisodes dramatiques.

Pour tenter d'y faire face, les groupes familiaux disposent au demeurant, compte tenu des informations, des compétences et des ressources dont ils disposent, d'une palette relativement peu diversifiée de réponses (Dupré et Guillaud 1999). La première mesure s'impose souvent comme une nécessité : elle vise à diminuer la ration journalière consommée (en ne prenant qu'un seul repas ou en préparant de plus petites quantités de mil) en réduisant, si besoin, la taille du groupe de commensalité. Le chef de ménage décide ainsi souvent de maintenir «en brousse » dans les hameaux de culture, les cadets sociaux et les dépendants (enfants en bas-âge inactifs, personnes âgées) jusqu'aux récoltes. Ils doivent y survivre en autarcie renforcée avec des conséquences nutritionnelles parfois désastreuses étant donné le surcroît de travail agricole saisonnier (semis et sarclage). Ce choix contraint va souvent de pair avec l'introduction de feuilles de cueillette, fraîches ou séchées, consommées seules ou mélangées à du mil et du sorgho tandis que le maïs fournit un appoint appréciable en fin de saison des pluies (septembre). Au fur et à mesure que les pression sur les ressources alimentaires s'accroît, ces aliments de substitution prennent une importance vitale: ils représentaient environ trente jours de consommation en 2001 et en 2002.

D'autres, moins nombreux, plus vulnérables ou moins entreprenants semblent davantage compter sur les relations traditionnelles d'échange (entraide, dons et contre-dons) au sein des grandes concessions pluri-familiales pour compenser les déficits saisonniers. En réalité, les dons «vrais », sont peu fréquents et réduits. La très grande majorité des transferts sont réalisés de manière indirecte: au sein des concessions rurales, les ménages en situation précaire (parents âgés, femmes répudiées, migrant de retour récent,...) sont pris en charge et intégrés temporairement au groupe de commensalité, au même titre que les bouviers peuls chargés de la garde et de l'entretien du troupeau. Cette redistribution représentait $15 \%$ des quantités consommées en 2001 et $11 \%$ en 2002.

En réalité, loin des idées reçues sur le caractère autarcique des économies enclavées, la survie alimentaire des communautés agropastorales passe très étroitement par l'acquisition de denrées supplémentaires, selon une hiérarchisation bien précise : la consommation ou la vente de produits de cueillette (karité, miel, néré, bois), la vente de biens alimentaires peu prisés (riz par exemple) ou la mobilisation de revenus issus d'activités saisonnières de complément (orpaillage, convoyage et gardiennage de bétail, petit commerce). Puis, si ces alternatives se révèlent insuffisantes, il optera pour une décapitalisation progressive, c'est-à-dire la vente d'actifs patrimoniaux au risque d'amoindrir sa capacité future à faire face au manque de nourriture selon un processus cumulatif (Pirau et al. 1996)

Tableau 4 : Répartition des recours alimentaires (en \%)

\begin{tabular}{|c|c|c|c|c|c|c|c|c|}
\hline & $\begin{array}{c}\text { Vente } \\
\text { de bétail }\end{array}$ & $\begin{array}{c}\text { Argent } \\
\text { économisé }\end{array}$ & $\begin{array}{c}\text { Aide } \\
\text { alimentaire }\end{array}$ & $\begin{array}{c}\text { Emprunt } \\
\text { financier }\end{array}$ & $\begin{array}{c}\text { Emprunt } \\
\text { céréalier }\end{array}$ & $\begin{array}{c}\text { Aide } \\
\text { financière }\end{array}$ & $\begin{array}{c}\text { Vente de } \\
\text { biens }\end{array}$ & Total \\
\hline 2001 & 33,9 & 25,0 & 20,3 & 8,2 & 5,1 & 5,1 & 2,3 & 100,0 \\
\hline 2002 & 36,4 & 25,6 & 12,9 & 11,1 & 8,0 & 3,7 & 2,1 & 100,0 \\
\hline
\end{tabular}

Source : IRD/Univ. Ouagadougou/IEDES-Paris I, 2001 et 2002. 
Dans cet espace agropastoral où cohabitent peul, mossi et gourmantché, l' «épargne sur pied » fournit la contribution la plus importante en période de disette, suivi par l'utilisation de l'épargne. Celle-ci provient essentiellement de la vente de produits agricoles (arachide, sésame, riz, sorgho rouge pour la bière, petits ruminants) ou d'une activité complémentaire de commerce de céréales et d'animaux. Quant à l'aide alimentaire, après avoir joué un rôle important en 2001 où la soudure était plus intense, elle diminuait très sensiblement l'année suivante. La vente de petits ruminants et de bovins représente ainsi près plus du tiers des recours dévelopés en période de soudure (tableau 4) et elle restait la tactique de court terme la plus fréquente ${ }^{12}$ quelle que soit la zone géographique pour les deux années de soudure. Signe de son importance économique et temporelle croissante, ce phénomène était étroitement corrélé avec les achats de mil et de sorgho (coefficients de $0.76^{* *}$ et $0.57 * *$ ) et avec le nombre de jours de soudure $\left(0.46^{* *}\right.$ et $\left.0.48^{* *}\right)$ en 2001 et 2002 .

A l'instar des ménages peuls dont l'essentiel des activités est traditionnellement tourné vers l'élevage, les ménages gourmantché de la Gnagna tendent de plus en plus à développer l'élevage en le combinant avec les cultures céréalières et vont jusqu'à pratiquer un élevage transhumant ou semi-transhumant en ayant recours aux compétences des bouviers peuls ${ }^{13}$. Des complémentarités étroites, sur la base de prestations contractuelles précises (travail contre céréales, lait contre céréales) sont souvent observées entre ces différentes communautés et sont susceptibles de participer efficacement à la lutte contre l'insécurité alimentaire conjoncturelle. Néanmoins, étant donné leur forte dépendance vis-à-vis de l'élevage bovin, leur relative insécurité foncière, la vulnérabilité des ménages peuls reste sensiblement supérieure. Progressivement, leur mobilité extrême - qui a constitué une forme reconnue d'adaptation aux contraintes bio-climatiques au cours des épisodes de grande sécheresse (1973-1974 et 1984-1985) - tend à devenir un élément de marginalisation socio-économique au fur et à mesure que l'enclavement se réduit et les échanges marchands prennent de l'importance. A contrario, les familles gourmantché peuvent jouer sur un plus grand nombre de recours en période de soudure, grâce à une plus large diversification d'activités rémunératrices (artisanat alimentaire, maraîchage, embouche ovine et bovine) et une meilleure insertion dans les projets de développement.

Néanmoins, la gestion combinée des greniers familiaux et des troupeaux constitue, pour la très grande majorité des agropasteurs, la meilleure réponse adverse au risque de disette. Elle est même stratégique dans la mesure où les autres modes de stockage, permettant une mutualisation renforcée des risques - greniers communautaires, stocks communs et banques de céréales - sont rares. Elle renvoie, de manière explicite, à la nature des régulations mises en œuvre : primat de la communauté sur l'individu, hiérarchies de statut et de genre. Dans ce domaine, les chefs de famille masculins disposent, en effet, nous l'avons déjà souligné, d'une sorte de pouvoir discrétionnaire redistributif. De multiples combinaisons s'offrent à eux après la récolte : décider ou reporter l'ouverture des greniers, utiliser en priorité les greniers individuels ou les greniers collectifs, associer consommation des céréales produites par le groupe familial et céréales achetées sur le marché. Ces différentes décisions sont d'abord prises en tenant compte du volume global et de la qualité des récoltes mais elles sont également conditionnées par l'existence d'éventuelles réserves antérieures (grenier «de garde ») et par le niveau d'une épargne mobilisable (monétaire ou « sur pied »). Au cours de l'année, les options de gestion peuvent être infléchies ou annulées, en fonction des possibilités d'achat ou de vente et des modifications éventuelles apportées à la consommation.

\footnotetext{
$1274 \%$ des ménages la pratiquaient en 2001 et $62 \%$ en 2002.

${ }^{13}$ Ainsi, l'élevage constitue la première ressource économique d'importance et place la province au quatrième rang national avec un peu plus de 6\% du cheptel bovin du pays (environ 290.000 têtes).
} 
L'analyse comparée des deux années de soudures permet de dresser une hiérarchie précise des modes de gestion les plus efficients pour faire face à la période de soudure, en fonction certes du niveau de disponibilités alimentaires mais surtout des anticipations d'achat ou de consommation (tableau 5). A quantité équivalente, ces dernières seront moins coûteuses pour l'agropasteur, compte tenu des bas prix céréaliers pratiqués après récolte et de la dépréciation marchande du bétail au cours de l'année alimentaire (qui court du mois de novembre au mois d'octobre suivant) en raison d'une offre plus abondante. Ainsi, des achats massifs anticipés (type 3) ou fractionnés (type 4) permettront de repousser la fin d'utilisation des greniers de plusieurs mois. La tactique la plus performante reste, malgré tout, le report d'utilisation des greniers et la consommation immédiate des achats réalisés (type 5). Néanmoins, à peine un ménage enquêté sur deux procédait à des anticipations, même de courte durée, avec des écarts importants selon les zones. Ainsi les anticipations d'achat sont d'autant plus importantes en volume et en durée que l'intensité des «risques apparents » est élevée. C'est pourquoi, les comportements des gestionnaires de greniers en zone 1 diffèrent sensiblement de ceux des autres zones. Cette hiérarchie entre zone de risque se retrouve également dans les différents indicateurs nutritionnels : la malnutrition protéino-énergétique est moins forte en zone 1, quel que soit la classe d'âge et le sexe (Janin et Martin-Prével 2003).

Tableau 5 : Types de gestion temporelle des greniers

\begin{tabular}{|c|c|c|c|c|c|}
\hline & Type 1 & Type 2 & Type 3 & Type 4 & Type 5 \\
\hline $\begin{array}{c}\text { Consommation des } \\
\text { greniers }\end{array}$ & Immédiate & Immédiate & Immédiate & Alternée & Reportée \\
\hline Achats céréaliers & Aucun & $\begin{array}{c}\text { Immédiats } \\
\text { après greniers }\end{array}$ & Anticipés & Fractionnés & Anticipés \\
\hline $\begin{array}{c}\text { Consommation des } \\
\text { achats }\end{array}$ & Aucune & Immédiate & Reportée & Alternée & Immédiate \\
\hline $\begin{array}{c}\text { Ajustement de la } \\
\text { ration en hivernage }\end{array}$ & Aucun & Baisse & $\begin{array}{c}\text { Baisse ou } \\
\text { hausse }\end{array}$ & $\begin{array}{c}\text { Baisse ou } \\
\text { hausse }\end{array}$ & $\begin{array}{c}\text { Aucun ou } \\
\text { hausse }\end{array}$ \\
\hline
\end{tabular}

La réponse la plus efficace en terme de risques consiste donc dans une bonne optimisation temporelle de la gestion des stocks. Celle-ci se révèle d'une grande complexité. Elle combine les reports d'utilisation de certains greniers, les achats anticipés de céréales sur les marchés (avant une hausse spéculative) afin d'assurer le plus haut niveau de sécurité alimentaire. Malgré tout, un «modèle» de gestion ayant permis de gérer efficacement la période de soudure passée peut se révéler inefficient l'année suivante dans la mesure où l'ensemble des paramètres alimentaires, économiques et sociétaux évoluent en permanence. Certains auteurs parlent ainsi de passage d'un « déficit subit » à un « déficit organisé » (Dupré et Guillaud 1999). En dépit de marges de manœuvre étroites, des prises de décision anticipés associées à une consommation prudente des disponibilités constituent une réponse probante dans un environnement aléatoire et incertain (Marchal 1990; Burton 1997). Ainsi, progressivement le caractère déterminant des contraintes bio-climatiques s'efface ; le risque d'insécurité alimentaire redevient le produit des rapports complexes entre société et nature tandis que la vulnérabilité alimentaire s'enracine dans les logiques parfois individuelles mais plus souvent communautaires des acteurs familiaux. 


\section{Conclusion}

Le choix de diversifier les échelles spatio-temporelles d'analyse s'est révélé essentiel pour aborder la question de l'insécurité alimentaire en milieu rural sahélien. En effet, d'importantes disparités spatiales ont été observées selon les méthodes adoptées et les niveaux de collecte des données : entre départements, entre villages mais également entre concessions d'un même village et même entre membres d'une même concession rurale, sans que la redistribution communautaire éventuelle (préparation commune en soudure, dons et contredons alimentaire) ne parvienne à les gommer. Qui plus est, à cette variabilité géographique viennent se surimposer des écarts significatifs de situation alimentaire d'une saison à l'autre. Il y a donc une nécessité réelle, pour les chercheurs comme pour les intervenants, à prendre en compte les différentes temporalités des risques et des acteurs. La recherche conduite justifie donc, de manière implicite, l'insatisfaction exprimée par les donateurs privés et institutionnels, puisqu'elle confirme que le ciblage géographique ou social de l'aide et, $a$ fortiori, l'identification des individus les plus vulnérables restent très difficiles à mettre en œuvre, dans un contexte d'information fragmentaire et d'instabilité géographique des populations rurales. Elle va néanmoins plus loin puisqu'elle propose des ouvertures méthodologiques et montre que les analyses géographiques ou sociales distanciées sont susceptibles d'introduire des biais importants dans la perception et la compréhension de l'insécurité alimentaire en milieu rural africain. Ce qui pose néanmoins la question de la capacité et de la volonté des intervenants à acquérir un corpus important de connaissances pour en dégager les indicateurs instantanés et complexes les plus pertinents pour la décision. De même que nous avons montré les biais importants d'une analyse basée sur les seuls indicateurs apparents de crise, communément utilisés par les décideurs, on peut s'interroger sur la pertinence de leurs choix méthodologiques pour l'analyse dynamique de la vulnérabilité $e^{14}$ alors même qu'ils conditionnent fortement les interventions.

Au-delà de ce que l'on peut apprendre sur les solutions et les parades développées par les communautés villageoises et les familles (Larivière et al. 1995 ; Pirau et al. 1996 ; Kinsey et al. 1998) pour faire face à l'insécurité alimentaire transitoire (i.e. à le gérer et à l'anticiper éventuellement) dans un environnement incertain et instable (Bebbington 1999), étudier la soudure alimentaire revient à tenir compte de l'ensemble des régulations mises en œuvre. Toute réflexion systémique sur l'insécurité alimentaire doit donc à la fois considérer les modalités de redistribution temporelle des ressources (cheptel, récoltes, argent) comme les modalités d'accès aux facteurs de production (terre, eau, travail, arbre) en fonction de la nature des régulations mises en place puisque la consommation alimentaire dépend encore essentiellement des denrées produites et stockées. Ce qui revient inévitablement à poser la question des rapports de pouvoir au sein des communautés villageoises (qui prend, par exemple, les décisions importantes engageant le groupe familial) puisqu'ils sont en mesure d'atténuer ou de renforcer les disparités individuelles d'accès aux aliments.

\section{Références bibliographiques}

Adger, W. N. 1999. Social vulnerability to climate change and extremes in coastal Vietnam. World Development $1999 ; 27(2): 249-269$.

\footnotetext{
${ }^{14}$ Relevés pluviométriques, recueil de prix sur les marchés, estimation rapide des revenus monétaires, taux de couverture des besoins céréaliers, indices patrimoniaux et de niveaux de vie, accessibilité aux infrastructures de base.
} 
Bebbington, A. 1999. Capitals and capacities : a framwork for analyzing peasant viability, rural livelihoods and poverty. World Development, 27 (12) : 2021-2044.

Burton, I. 1997. Vulnerability and adaptative response in the context of climate and and climate change, Climatic Change, 36 (1-2) : 185-196.

Courade, G. 2001. Paupérisation et inégalités d'accès aux ressources. In : Winter G. coord. Inégalités et politiques publiques en Afrique. Pluralité des normes et jeux d'acteurs. 2001 Paris, Karthala : 25-39.

Dupré, G. et Guillaud, D. 1999. Entre incertitude et sécurité : les systèmes de production en Aribinda (Burkina Faso). In : Champaud J. et Poncet Y. éds. Les temps du Sahel, Paris, IRD : 109-130.

Egg, J. et Gabas, J.-J. 1997. La prévention des crises alimentaires au Sahel et le rôle des dispositifs d'information. Paris, Stateco, 87-88: 5-20.

Gado B. A. 1993. Une histoire des famines au Sahel : études des grandes crises alimentaires $\left(X I X^{\circ}-X X^{\circ}\right.$ siècles). L'Harmattan, Col. Racines du présent, Paris, 200 p.

Hamelin A.-M., Beaudry M. et Habicht J.-P. 1998. La vulnérabilité des ménages à l'insécurité. in : La quête de la sécurité alimentaire au $21^{e}$ siècle, Revue canadienne d'études du développement, Université d'Ottawa, $19: 278-306$.

Janin, P. 2001a. «L'insécurité alimentaire rurale en Côte d'Ivoire : une réalité cachée, aggravée par la société et le marché », Paris, John Libbey Eurotext, Cahiers Agricultures, 10 (4) : 233-241.

Janin, P. 2001b. Proposition de zonage géographique de la Gnagna (Burkina Faso). UR 106, Centre IRD, Ouagadougou, $10 \mathrm{p}$.

Janin, P. et Cambrézy L. 2003. «Le risque alimentaire en Afrique », pp. 88-103, in : Veyret (Y.) éd., 2003. - Les risques, Paris, Col. Dossiers des images économiques du monde (DIEM), SEDES, 255 p.

Janin, P. et Martin-Prével Y. 2003. «Disponibilités alimentaires, niveaux de risque apparent et état nutritionnel en milieu rural sahélien burkinabé », $2^{\text {ème }}$ Atelier International Voies alimentaires d'amélioration des situations nutritionnelles en Afrique de l'Ouest : Le rôle des technologues alimentaires et des nutritionnistes, Ird-FAO-Université de Wageningen, Ouagadougou (23-28 novembre 2003), 15 p.

Jaspers, S. and Shoham, J. 1999. Targeting the vulnerable : a review of the necessity and feasability of targeting vulnerable households. Disasters, 23 (4) : 359-372.

Kinsey, B. Burger, K. and Gunning, J. W. 1998. Coping with drought in Zimbabwe : survey evidence on responses of rural households to risk, World Development, 26 (1) : 89-110.

Larivière, S., Martin, F. et Savadogo, K. 1995. Stratégies des ménages ruraux en matière de sécurité alimentaire dans un contexte d'ajustement structurel : le cas de la province du Passoré au Burkina-Faso. Grenoble, PUG, Économies et sociétés (série Développement agro-alimentaire), AG 22 (3-4) : 145-165.

Makki, S. S., Tweeten, L. G. and Miranda, M. J. 2001. Storage-trade interactions under uncertainty. Implications for food security. Journal of Policy modeling, 23 : 127-140.

Marchal, J.-Y. 1990. En Afrique soudano-sahélienne : la course contre le temps. In : Eldin, M. et Milleville, P. éds. Le risque en agriculture, Paris, ORSTOM, Col. A travers champs : 225-267.

Moseley, W. G. 2001. African evidence on the relation of poverty, time preference and the environment. Ecological Economics, 38 : 317-326.

Ouedraogo, F. de C. 2001. Atlas de la vulnérabilité alimentaire, Ouagadougou, IRD/Université de Ouagadougou, $150 \mathrm{p}$.

Pirau, M., Buldgen, A., Drugmant, F., Fall, M. et Compère, R. 1996. Adaptation des stratégies paysannes aux risques climatiques et à la pression démographique en zone soudano-sahélienne, Paris, John Libbey Eurotext, Aupelf-Uref, Cahiers d'études et de recherches francophones (Agricultures), 5 : 99-108.

Raynaut, C. dir. 1998. Sahels. Diversité et dynamiques des relations sociétés-nature. Paris, Karthala, Col. Hommes et sociétés, $430 \mathrm{p}$.

Reardon, T. and Matlon, P. 1989. Seasonal food insecurity and vulnerability in drought-affected regions of Burkina Faso. In : Sahn D. Seasonal variation in the Third World agriculture, John Hopkins University Press, Baltimore : 118-136.

Reardon, T. and Taylor, J. E. 1996. Agroclimatic shocks, income inequality and poverty : an evidence from Burkina Faso, World Development, 24 (5) : 901-914.

Ribot, J. C., Magalhães, A.R., and Panagides, S. S. éds. 1996. Climate variability, climate change and social vulnerability in the semi-arid Tropics, Cambridge Press University, Cambridge, : p. 